Received: 21 September 2016 Accepted: 7 April 2017 Published online: 12 May 2017
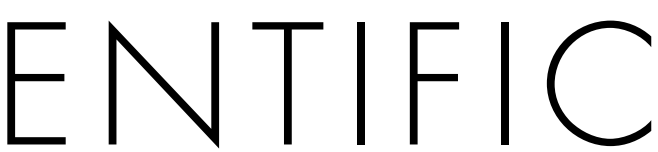

REP
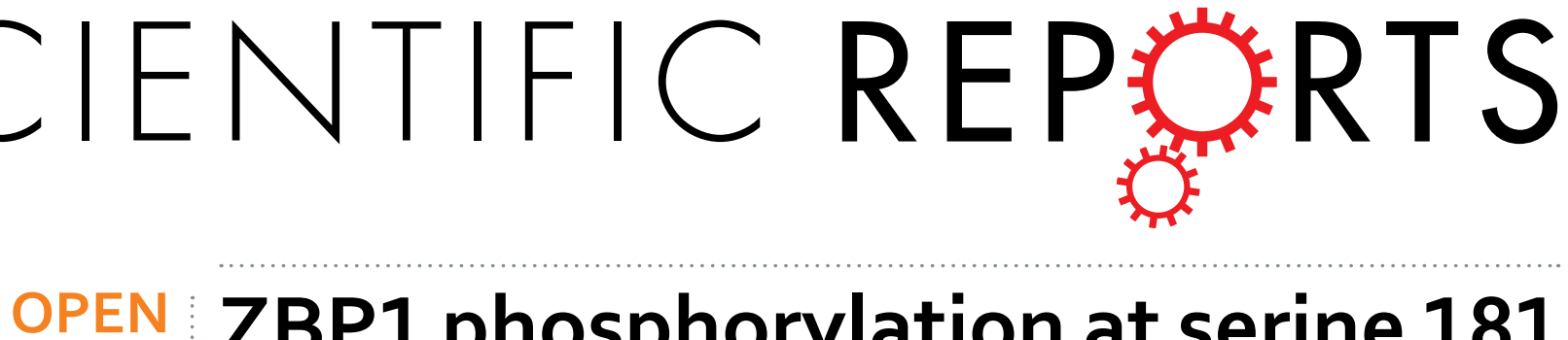

\section{ZBP1 phosphorylation at serine 181 regulates its dendritic transport and the development of dendritic trees of hippocampal neurons}

Anna S. Urbanska ${ }^{1}$, Aleksandra Janusz-Kaminska ${ }^{1}$, Katarzyna Switon ${ }^{1}$, Alicia L. Hawthorne ${ }^{2}$, Malgorzata Perycz ${ }^{1}$, Malgorzata Urbanska ${ }^{1}$, Gary J. Bassell ${ }^{2}$ \& Jacek Jaworski ${ }^{1}$

Local protein synthesis occurs in axons and dendrites of neurons, enabling fast and spatially restricted responses to a dynamically changing extracellular environment. Prior to local translation, mRNA that is to be translated is packed into ribonucleoprotein particles (RNPs) where RNA binding proteins ensure mRNA silencing and provide a link to molecular motors. ZBP1 is a component of RNP transport particles and is known for its role in the local translation of $\beta$-actin mRNA. Its binding to mRNA is regulated by tyrosine 396 phosphorylation, and this particular modification was shown to be vital for axonal growth and dendritic branching. Recently, additional phosphorylation of ZBP1 at serine 181 (Ser181) was described in non-neuronal cells. In the present study, we found that ZBP1 is also phosphorylated at Ser181 in neurons in a mammalian/mechanistic target of rapamycin complex 2-, Src kinase-, and mRNA binding-dependent manner. Furthermore, Ser181 ZBP1 phosphorylation was essential for the proper dendritic branching of hippocampal neurons that were cultured in vitro and for the proper ZBP1 dendritic distribution and motility.

Local protein synthesis is a phenomenon of regulated translation that occurs in subdomains of a cell, often distant from the major sites of protein production (e.g., leading edges of fibroblasts and neuronal axonal growth cones and dendrites). It provides a means of local and transient cell polarization and enables fast responses to local stimuli. Neurons have been the most intensely studied because of their importance for local protein synthesis. In these cells, local protein synthesis regulates axonal growth cone navigation, dendritic arbor and spine formation, and synaptic plasticity ${ }^{1-3}$. Local translation requires the transportation of translationally dormant mRNA to the destination point, often over relatively long distances, where mRNA becomes derepressed and translationally competent. mRNAs that are to be locally synthesized contain tagging sequences that are recognized and bound by protein complexes that provide both translational silencing and attachment to molecular motors (i.e., kinesins, dyneins, and myosins). These so-called ribonucleoprotein particles (RNPs) can be transported along microtubules and microfilaments, or they may stall at destination points while awaiting a signal to release bound mRNA for translation ${ }^{4}$. In neurons, changes in RNP dynamics and the translational unsilencing of mRNA can occur very rapidly, often in response to extracellular stimuli (e.g., trophic factors, calcium influx, or neurotransmitters) and are controlled by the phosphorylation of RNP proteins ${ }^{5-7}$.

Zipcode binding protein 1 (ZBP1; also known as IMP1 or IGF2BP1) is an mRNA binding protein that is found in RNPs. ZBP1 was described as $\beta$-actin mRNA-binding protein, which ensures its proper cellular distribution and translational silencing. In neurons, the local synthesis of $\beta$-actin, controlled by ZBP1 and its orthologs (e.g., IMP1 and Vg1 RBP), contributes to axonal growth cone navigation, proper dendritic arborization, and dendritic spine formation ${ }^{8-12}$. The ability of ZBP1 to silence $\beta$-actin mRNA is dynamically regulated by phosphorylation. In response to trophic factors, ZBP1 is phosphorylated at tyrosine 396 (Tyr396) by Src and releases $\beta$-actin mRNA for translation ${ }^{5,6,13}$. In fact, proper axonal growth cone navigation and dendritic branching both require the ability of ZBP1 to be phosphorylated at Tyr3965,6,9. Recently, additional phosphorylation sites of ZBP1 and its orthologs were described. For example, in non-neuronal cells, serine 181 (Ser181) of IMP1 is cotranslationally

${ }^{1}$ International Institute of Molecular and Cell Biology, Warsaw, Poland. ${ }^{2}$ Department of Cell Biology, Emory University School of Medicine, Atlanta, GA, USA. Aleksandra Janusz-Kaminska and Katarzyna Switon contributed equally to this work. Correspondence and requests for materials should be addressed to J.J. (email: jaworski@iimcb.gov.pl) 


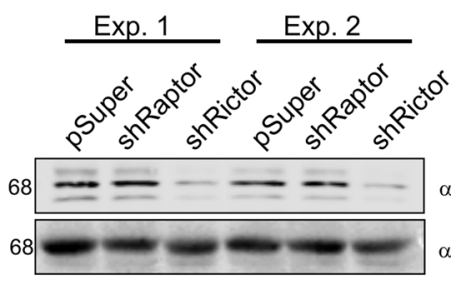

d
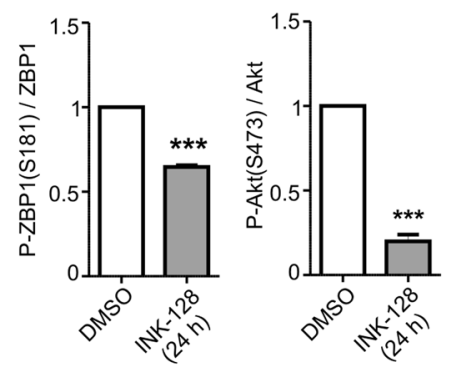

b

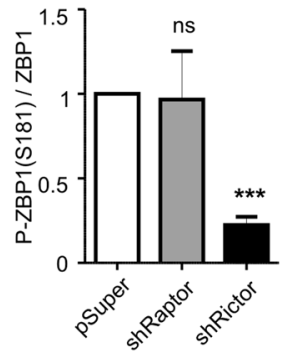

C

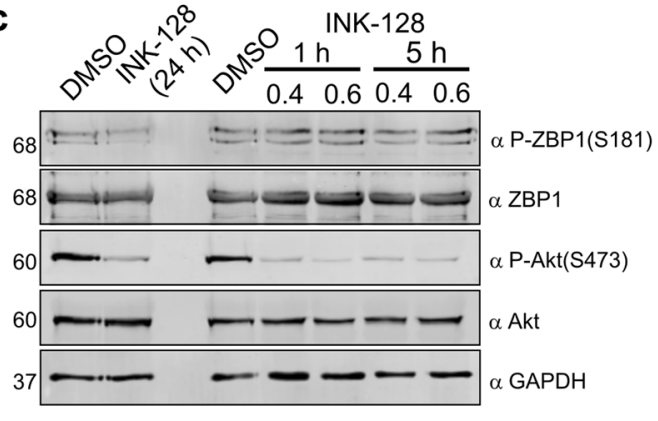

e
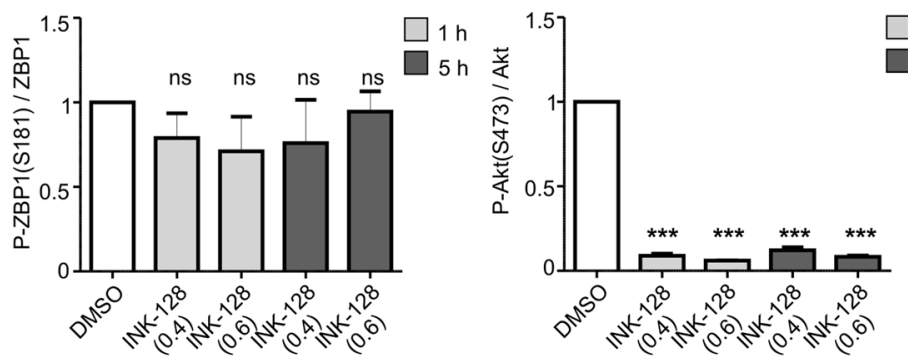

Figure 1. ZBP1 is phosphorylated at Ser181 in neurons in an mTORC2-dependent manner. (a) Western blot analysis of levels of ZBP1 phosphorylated at Ser181 (P-ZBP1[S181]) and total ZBP1 in protein lysates obtained from DIV8 neurons nucleofected on DIV0 with empty pSuper (control vector) or pSuper that encoded shRNA against Raptor or Rictor. Cropped blots are presented (for full length blots see Supplementary Information). (b) Results of quantitative Western blot analysis. The data are presented as a ratio of the signal intensity of P-ZBP1(S181) to the signal intensity of ZBP1 \pm SEM. $* * * p<0.001, n s$ - non significant (One sample $t$-test). Number of independent experiments $(N=4)$. (c) Western blot analysis of levels of P-ZBP1(S181), total ZBP1, P-Akt(S473) and total Akt in protein lysates obtained from DIV10 neurons treated for 1, 5 or $24 \mathrm{~h}$ with vehicle (DMSO) or INK128 at indicated concentrations. GAPDH is shown as an additional loading control. Cropped blots are presented (for full length blots see Supplementary Information). (d) Results of quantitative Western blot analysis. The data are presented as a ratio of the signal intensity of P-ZBP1(S181) to ZBP1 or P-Akt to Akt \pm SEM. $* * * p<0.001, n s$ - non significant (One sample $t$-test). Number of independent experiments $(N=3)$.

phosphorylated by mammalian/mechanistic target of rapamycin complex 2 (mTORC2) and protected by RNA binding. In mouse embryonic fibroblasts and RD rhabdomyosarcoma cells, Ser181 ZBP1 phosphorylation is needed for proper splicing and the cap-independent translation of insulin growth factor 2 (IGF2) mRNA. However, to date, the presence of Ser181 ZBP1 phosphorylation and its regulation in neurons and significance for neuronal development have not been tested.

The present study showed that ZBP1 is stably phosphorylated at Ser181 in neurons. This phosphorylation was mTORC2- and mRNA binding-dependent. Moreover, we found less ZBP1 with phosphorylated Ser181 in cells with increased Src activity. Furthermore, rescue experiments with ZBP1 short-hairpin RNA (shRNA) and a ZBP1 mutant that mimicked non-phosphorylated ZBP1 $\left(\mathrm{ZBP}_{\mathrm{S} 181 \mathrm{~A}}\right)$ provided evidence that Ser181 ZBP1 phosphorylation was critical for the proper dendritic branching of hippocampal neurons that were cultured in vitro. Finally, we found that Ser181 phosphorylation is needed for proper ZBP1 mobility and dendritic distribution. Taken together these findings uncover the role of Ser181 phosphorylation in ZBP1 trafficking and dendritic development.

\section{Results}

ZBP1 is phosphorylated at Ser181 in neurons in an mTORC2-dependent manner. ZBP1 is phosphorylated at Ser181 in non-neuronal cells ${ }^{14}$, but this phosphorylation was not previously investigated in neurons. To verify the existence of such phosphorylation in neurons, we used a custom-made antibody that was raised against Ser181-phosphorylated rat ZBP1. This antibody detected, in an alkaline phosphatase-dependent manner, green fluorescent protein (GFP)-tagged wildtype rat ZBP1 ( 96kDa) but not ZBP1 with Ser181 mutated to alanine (S181A) that was overexpressed in Neuro2a cells (Supplementary Fig. S1). Both proteins were recognized by an antibody that detects ZBP1 regardless of its phosphorylation status (Supplementary Fig. S1). The new phospho-specific antibody detected an endogenous protein of a predicted size $(\sim 68 \mathrm{kD})$ in lysates of developing rat neurons that were cultured in vitro and lysates of hippocampi and cortices of embryonic day 17 (E17) wildtype mice (Fig. 1a, Supplementary Fig. S1). No such band was detected in protein lysates that were obtained from hippocampi and cortices of E17 IMP1 $1^{-1-}$ mice $^{15}$ (Supplementary Fig. S1), further supporting the specificity of the tested antibody.

Next, we tested whether mTORC2 contributes to ZBP1 phosphorylation in neurons similarly to the way it does in non-neuronal cells ${ }^{14}$. Freshly isolated rat embryonic cortical neurons were nucleofected with pSuper (control plasmid) or pSuper that encoded previously validated shRNA against Raptor or Rictor (i.e., components of mTORC1 and mTORC2, respectively ${ }^{16,17}$; Supplementary Fig. S2). The analysis of lysates from 8 days 


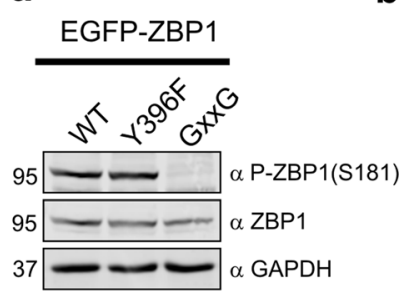

b

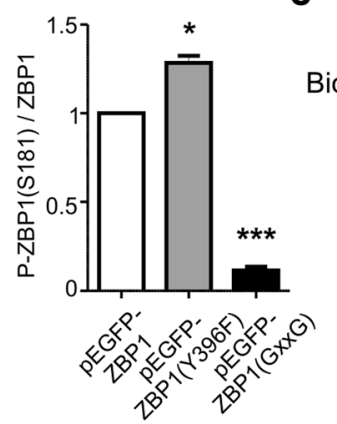

C

$$
\text { SrC-HA WT CA KD }
$$

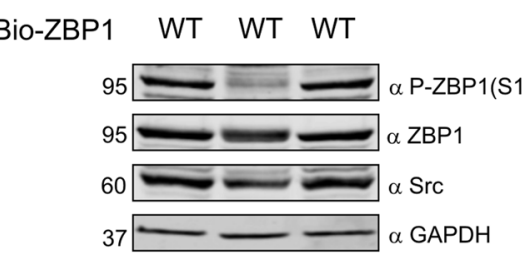

d

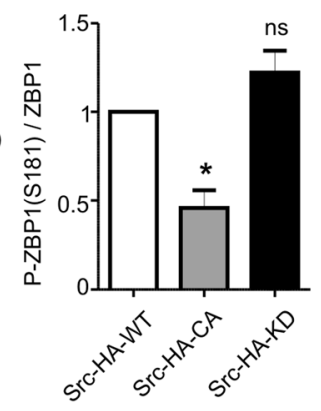

Figure 2. Serine 181 phosphorylation is dynamically regulated by Src activity. (a) Western blot analysis of levels of ZBP1 phosphorylated at Ser181 (P-ZBP1[S181]) and total ZBP1 in protein lysates obtained from Neuro2a cells transfected with pEGFP-ZBP1, pEGFP-ZBP $1_{\mathrm{Y} 396 \mathrm{~F}}$, and $\mathrm{pEGFP-ZBP} 1_{\mathrm{GxxG}}$. GAPDH is shown as an additional loading control. Cropped blots are presented (for full length blots see Supplementary Information). (b) Results of quantitative Western blot analysis. The data are presented as a ratio of the signal intensity of P-ZBP1(S181) to the signal intensity of ZBP1 \pm SEM. ${ }^{*} p<0.05$, *** $p<0.001$ (One sample $t$-test). Number of independent experiments $(N=3)$. (c) Western blot analysis of levels of ZBP1 phosphorylated at Ser181 (P-ZBP1[S181]), total ZBP1, and Src in protein lysates obtained from Neuro2a cells transfected with pBIO-ZBP1 and plasmids that encoded $\mathrm{Src}_{\mathrm{WT}}, \mathrm{Src}_{\mathrm{CA}}$, or $\operatorname{Src}_{\mathrm{DN}}$. GAPDH is shown as an additional loading control. Cropped blots are presented (for full length blots see Supplementary Information). (d) Results of quantitative Western blot analysis. The data are presented as a ratio of the signal intensity of P-ZBP1(S181) to the signal intensity of ZBP $1 \pm$ SEM. ${ }^{*} p<0.05, n s$ - non significant (One sample $t$-test). Number of independent experiments $(N=4)$.

in vitro (DIV8) electroporated neurons indeed revealed a decrease in P-ZBP1(S181)/ZBP1 ratio in cells with Rictor knockdown compared with Raptor knockdown and control cells (Fig. 1a,b). To further confirm the role of mTORC2 in controlling Ser181 ZBP1 phosphorylation in neurons and gain further insight into the dynamics of this process, we treated DIV10 neurons with Ink128, an ATP-competitive inhibitor of mTOR, for 1, 5 or $24 \mathrm{~h}$. mTOR inhibition for $24 \mathrm{~h}$ was sufficient to decrease Ser181 ZBP1 phosphorylation (Fig. 1c,d). In contrast, shorter treatments resulted in no difference between treated and untreated cells, although both inhibitors led to the loss of Ser473 phosphorylation of Akt, a prototypical mTORC2 substrate (Fig. 1c,e). These results show that mTORC2 contributes to Ser181 ZBP1 phosphorylation, which is rather stable under basal culture conditions.

Ser181 phosphorylation is regulated in response to Src-dependent ZBP1 modification. Next, we tested under what additional conditions the phosphorylation of ZBP1 at Ser181 can change in neural cells. In non-neuronal cells, Ser181 is dephosphorylated upon the release of bound mRNA ${ }^{14}$. Consistent with this finding, a ZBP1 mutant with decreased ability to bind mRNA $\left(\mathrm{ZBP} 1_{\mathrm{GXXG}}\right)$ was poorly phosphorylated at Ser 181 when it was overexpressed in Neuro2a cells compared with wildtype ZBP1 (Fig. 2a,b). In neurons, the release of $\beta$-actin mRNA from ZBP1 RNPs is dynamically regulated by the Src-driven phosphorylation of Tyr $396^{5}$. Thus, the ZBP1 mutant, which cannot be phosphorylated by $\operatorname{Src}\left(\mathrm{ZBP} 1_{\mathrm{Y} 396 \mathrm{~F}}\right)$, should less efficiently release bound mRNA and be more phosphorylated at Ser181. Indeed, as opposed to $\mathrm{ZBP} 1_{\mathrm{GXXG}}, \mathrm{ZBP} 1_{\mathrm{Y} 396 \mathrm{~F}}$ was slightly, although significantly, more phosphorylated at Ser181 compared with wildtype protein when it was overexpressed in Neuro2a cells (Fig. 2a,b). The phosphorylation of ZBP1 at Ser181 significantly decreased when ZBP1 was cotransfected to Neuro2a with constitutively active Src kinase but not its kinase-dead variant (Fig. 2c,d). Tyr396 ZBP1 phosphorylation was absent in cells that were transfected with $\mathrm{Src}^{\mathrm{KD}}$, whereas it was present in cells that overexpressed $\mathrm{Src}^{\mathrm{CA}}$ (Supplementary Fig. S3). Notably, wildtype and phospho-mutants (S181A, S181E [phospho-mimicking mutant]) of ZBP1 were phosphorylated at Tyr396 by Src to the same extent (Supplementary Fig. S3). This suggests that ZBP1 Src-dependent phosphorylation and subsequent mRNA release may regulate Ser181 phosphorylation. On the other hand, Ser181 phosphorylation does not prevent Tyr396 ZBP1 phosphorylation by Src. Altogether, our observations suggest that Ser181 phosphorylation in neurons is relatively stable but can change in response to Src-dependent ZBP1 phosphorylation.

ZBP1 Ser181 phosphorylation is critical for the proper dendritic arborization of hippocampal neurons. Next, we investigated whether Ser181 phosphorylation is meaningful for one of the neuronal functions of ZBP1. We previously found that ZBP1 was critical for the proper pattern of dendritic branching of hippocampal neurons that were cultured in vitro ${ }^{9}$. Thus, we examined the contribution of Ser181 ZBP1 phosphorylation to the proper development of dendritic arbors. First, we confirmed in Neuro2a cells that S181 mutations either to A or E do not affect ZBP1 stability (Supplementary Fig. S4). Next, we used the same approach as previously described ${ }^{9}$, in which we assayed $\mathrm{ZBP} 1_{\mathrm{S} 181 \mathrm{~A}}$ and $\mathrm{ZBP} 1_{\mathrm{S} 181 \mathrm{E}}$ mutants with regard to their ability to rescue dendritic arbor simplification that was induced by ZBP1 knockdown. DIV7 hippocampal neurons were transfected with a plasmid that encoded validated ZBP1 shRNA (pSuper-ZBP1sh) and a plasmid that encoded an RNA interference-resistant wildtype, S181A or S181E EGFP-ZBP1 mRNA (EGFP-ZBP1*, EGFP-ZBP1* ${ }_{\text {S181A }}$, and EGFP-ZBP1* $\left.{ }_{\text {S181E }}\right)$. As an additional control, we used cells that were transfected with pSuper-ZBP1sh and 

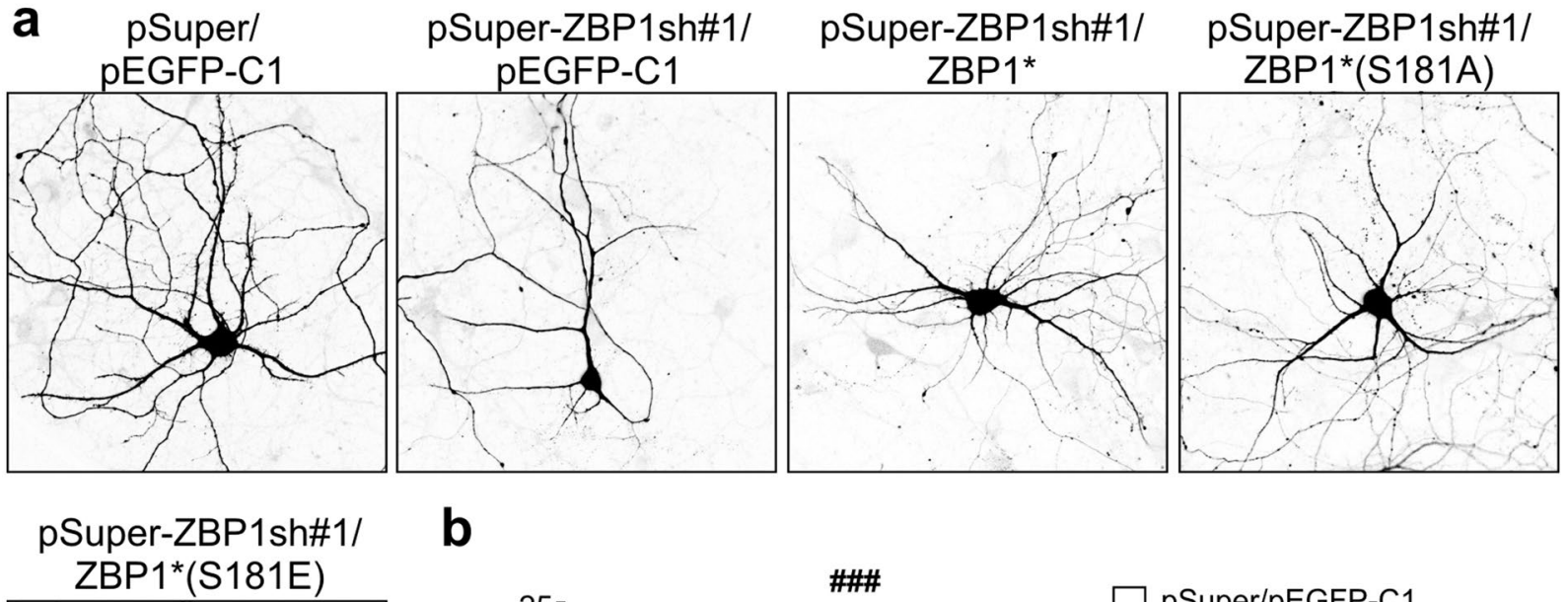

b
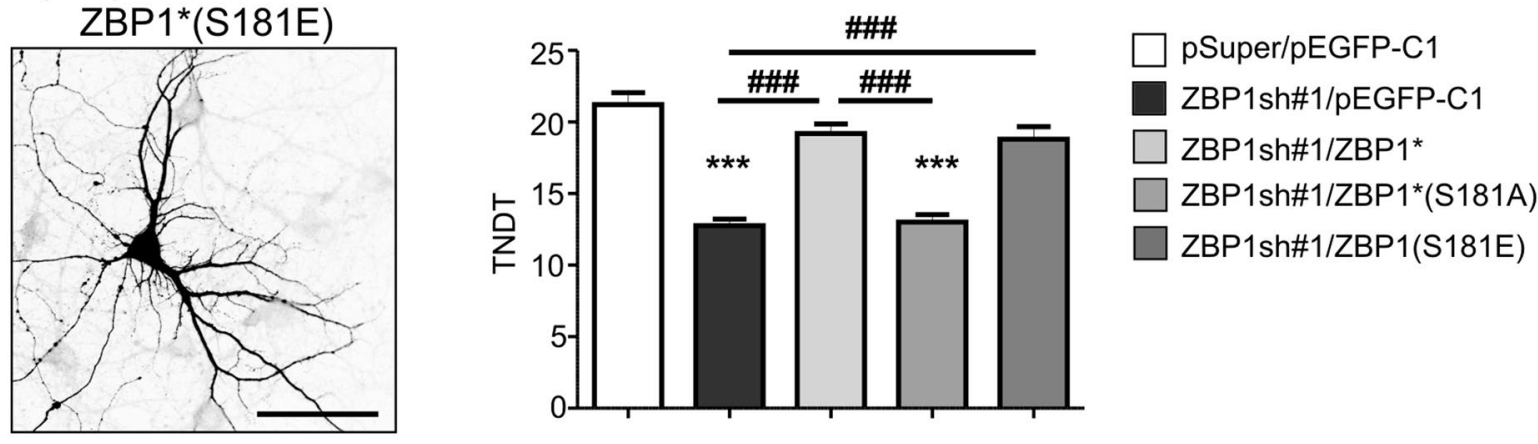

Figure 3. ZBP1 phosphorylation at Ser181 is needed for the proper dendritic arborization of hippocampal neurons. (a) Representative confocal images of cultured hippocampal neurons transfected on DIV8 for 2 days as indicated. Neuron morphology was visualized by co-transfection with mRFP. Scale bar $=50 \mu \mathrm{m}$. (b) Total number of dendritic tips (TNDT) of neurons transfected as in (a). The data are expressed as mean $\pm \mathrm{SEM}$. $* * * p<0.001$, compared with pSuper/pEGFP-C1-transfected cells; ${ }^{\# \#} p<0.001$, compared as indicated (Kruskal-Wallis test followed by Dunn's post hoc test). Cell images were obtained from two independent culture batches. Number of cells per variant: pSuper/pEGFP-C1 $(n=35), \mathrm{ZBP} 1 \mathrm{sh} \# 1 / \mathrm{pEGFP}-\mathrm{C} 1(n=36)$, ZBP1sh\#1/ pEGFP-ZBP1* $(n=35), Z B P 1$ sh\#1/pEGFP-ZBP1 ${ }_{\text {s181A }}(n=55)$, and ZBP1sh\#1/pEGFP-ZBP1* ${ }_{\text {s181E }}(n=47)$.

EGFP. After 3 days, the total number of dendritic tips (TNDT) was analyzed. As previously reported, an shRNA against ZBP1 resulted in a significant decrease in the TNDT, and co-transfection of EGFP-ZBP1* fully rescued this defect (Fig. 3). However, when EGFP-ZBP1* ${ }_{\text {s181A }}$ was transfected instead, we did not observe TNDT rescue. The cotransfection of EGFP-ZBP $1{ }^{*}{ }_{\text {S181E }}$ fully rescued the knockdown phenotype but did not cause any further increase in the TNDT. This observation is consistent with a previous suggestion that the majority of cellular ZBP1 is phosphorylated at Ser $181^{14}$. However, when Y396F mutation was introduced to ZBP $1_{\mathrm{S} 181 \mathrm{E}}$, the double mutant lost the ability to rescue phenotypic effects of ZBP1 knockdown, similar to ZBP1Y396F (Supplementary Fig. S5). Thus, we concluded that the phosphorylation of ZBP1 at Ser181 is involved in the control of proper neuronal dendritic morphology but its role might be secondary to phosphorylation of tyrosine 396 of ZBP1.

Ser181 phosphorylation is required for the proper dendritic distribution of ZBP1. In our previous work, we found that ZBP1 is differentially distributed in dendrites of neurons that are actively growing their dendritic arbors compared with mature dendrites. This suggested that proper dendritogenesis requires accurate ZBP1 distribution, namely the concentration of ZBP1 at dendrite branching points, which may correspond to hot spot of local synthesis of $\beta$-actin and other proteins needed for dendritic growth and branching. Accordingly, we tested whether Ser181 ZBP1 phosphorylation contributes to the spatial dendritic distribution of this protein. For more accurate quantitative analysis, we designed plasmids that simultaneously expressed EGFP-ZBP1 (or S181A and S181E mutants) and fluorescent protein - tdTomato (Supplementary Fig. S6) to correct for effects of unequal transfection of individual neurons. Next, the spatial distribution of EGFP-ZBP1 and its mutants in dendrites of DIV10 neurons was quantitatively analyzed. Wildtype ZBP1 concentrated at dendritic branching points as described previously ${ }^{9}$ (Fig. $4 \mathrm{a}$ ). $\mathrm{ZBP}_{\mathrm{S} 181 \mathrm{E}}$ displayed a very similar distribution (Fig. $4 \mathrm{c}$ ). In contrast, $\mathrm{ZBP} 1_{\mathrm{S} 181 \mathrm{~A}}$ was almost equally distributed between branching points and interbranching dendritic segments (Fig. 4b). Comparisons of the normalized EGFP fluorescence ratio in a dendritic D2 segment to the following R2 branching point (Fig. 4) of ZBP1- and $\mathrm{ZBP} 1_{\mathrm{S} 181 \mathrm{~A}}$-expressing neurons revealed a statistically significant difference (EGFP-ZBP1 [ $n=34]: 0.53 \pm 0.05$; EGFP-ZBP1 $1_{\text {S181A }}[n=32]: 0.88 \pm 0.09 ; p=0.0014$, Mann-Whitney test), confirming that $\mathrm{ZBP} 1_{\mathrm{S} 181 \mathrm{~A}}$ was more equally distributed and not enriched at dendritic branch points.

Changed distribution of $Z \mathrm{ZPP} 1_{\mathrm{S} 181 \mathrm{~A}}$ theoretically could be a result of its inability to bind $\mathrm{mRNA}$ as it was shown for $\mathrm{ZBP} 1_{\mathrm{GXXG}}$ mutant. Thus, we tested the ability of $\mathrm{ZBP} 1_{\mathrm{S} 181 \mathrm{~A}}$ to bind $\beta$-actin $\mathrm{mRNA}$, a canonical target of $\mathrm{ZBP} 1$. 
a

tdTomato

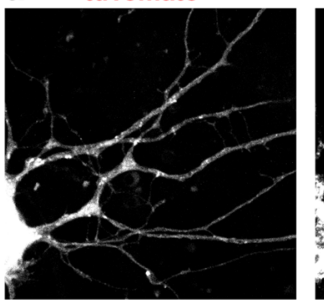

b
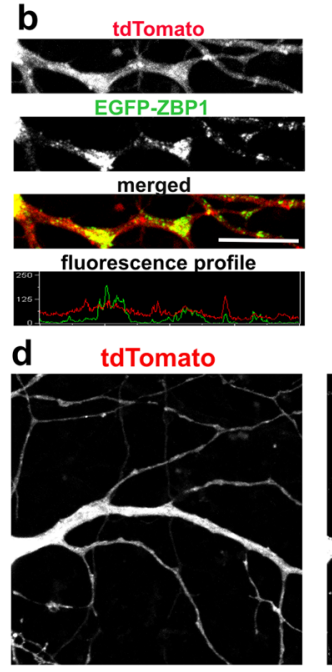

e

tdTomato

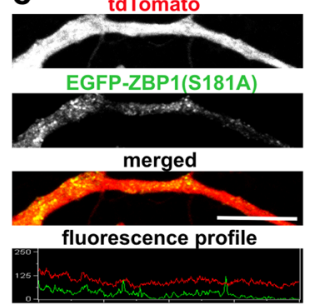

g tdTomato

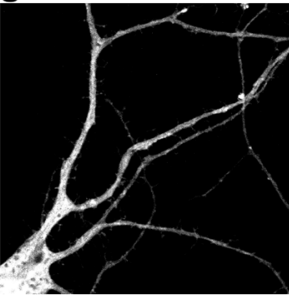

h

tdTomate

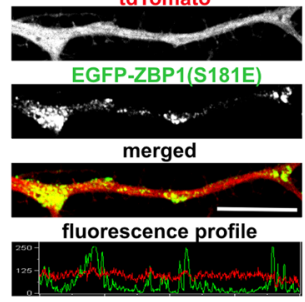

EGFP-ZBP1

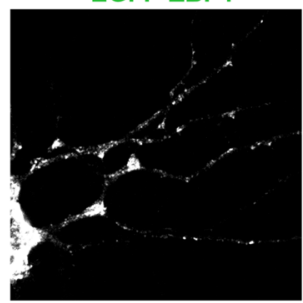

C
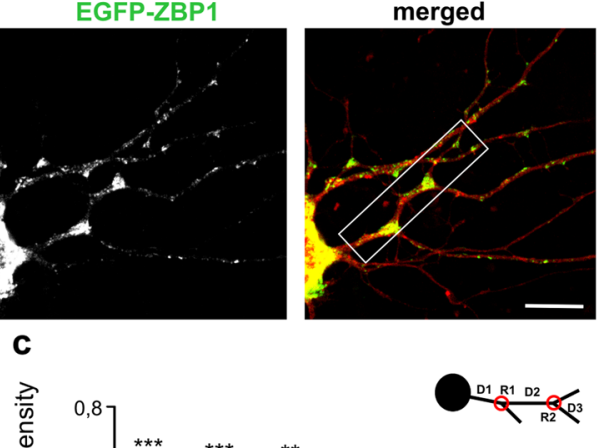

$\frac{102}{{ }_{22}^{2}} 23$

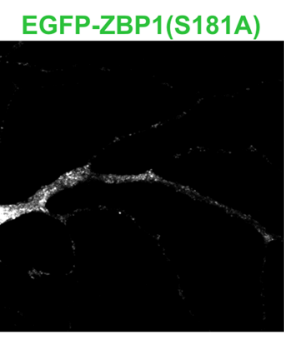

f

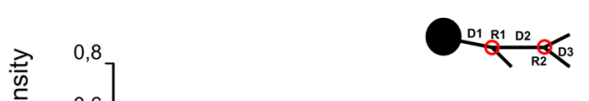

EGFP-ZBP1(S181A)

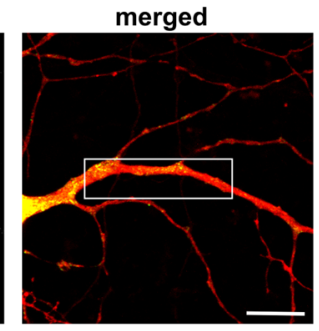

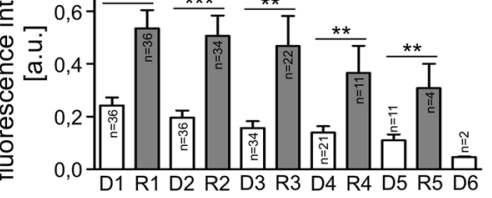

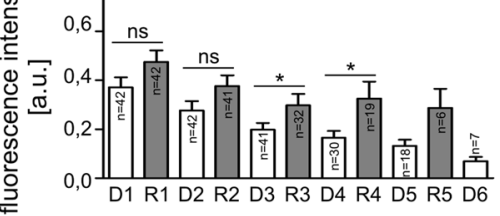

0,0 D1 R1 D2 R2 D3 R3 D4 R4 D5 R5 D6
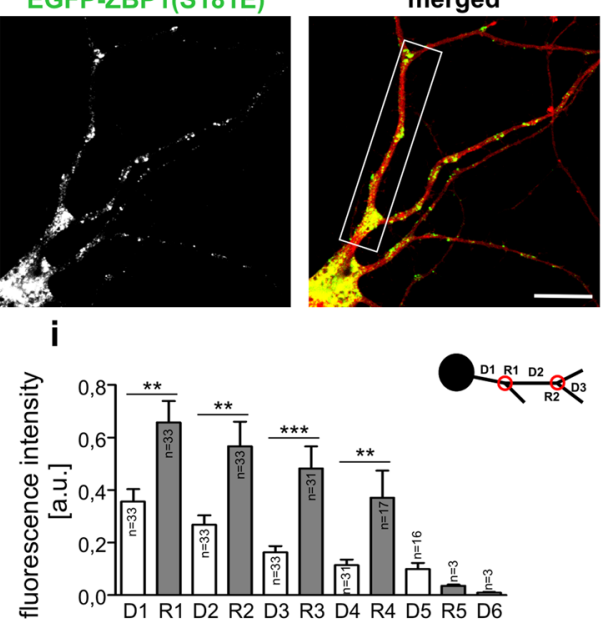

Figure 4. ZBP1 phosphorylation at Ser 181 is needed for its proper dendritic distribution. Representative confocal images of hippocampal neurons transfected on DIV7 with pEGFP-ZBP1-P2A-tdTomato (a), pEGFP$\mathrm{ZBP}_{\mathrm{S}_{181 \mathrm{~A}}}-\mathrm{P} 2 \mathrm{~A}$-tdTomato $(\mathbf{d})$, or pEGFP-ZBP1 $1_{\mathrm{S} 181 \mathrm{E}}-\mathrm{P} 2 \mathrm{~A}$-tdTomato $(\mathbf{g})$. Expression proceeded for $30 \mathrm{~h}$. (b,e,h) Representative micrographs of single dendrites of transfected cells with corresponding profiles of fluorescence. $(\mathbf{c}, \mathbf{f}, \mathbf{i})$ Normalized mean intensities of EGFP-ZBP1 fluorescence \pm SEM measured at dendritic branching points $(\mathrm{R})$ and interbranching dendritic segments (D). The data were obtained from three independent neuronal cultures. The data are expressed as mean \pm SEM. $* * * p<0.001, * * p<0.01, * p<0.05$, ns - non-significant. Student's $t$-test. Number of cells per variant: pEGFP-ZBP1-P2A-tdTomato $(n=36)$, pEGFP-ZBP1(S181A)-P2AtdTomato $(n=42)$, pEGFP-ZBP1(S181E)-P2A-tdTomato $(n=33)$. Number of analyzed branches for each order is provided in each graph. 
a

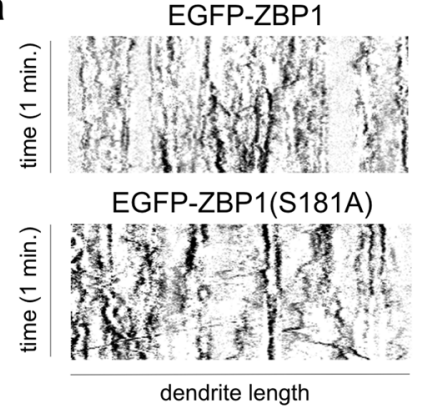

d

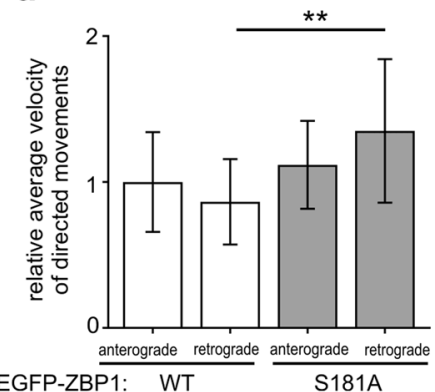

b

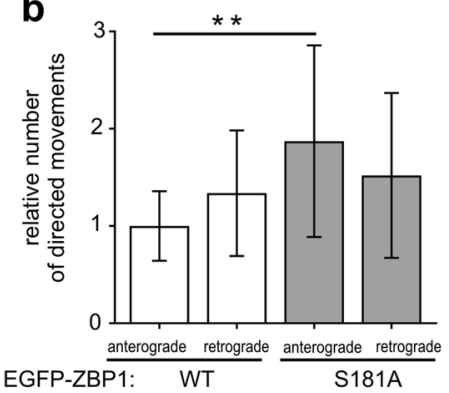

e

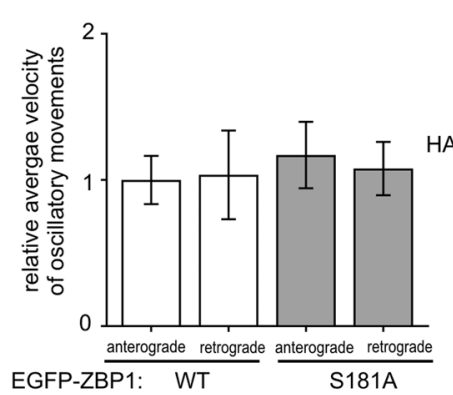

C

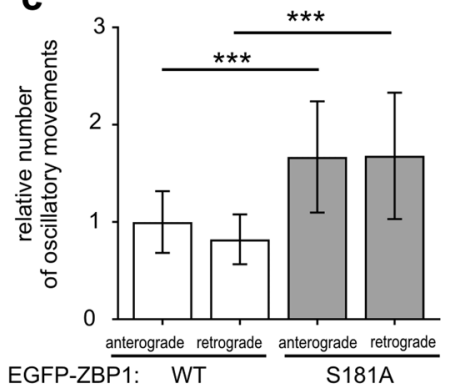

f

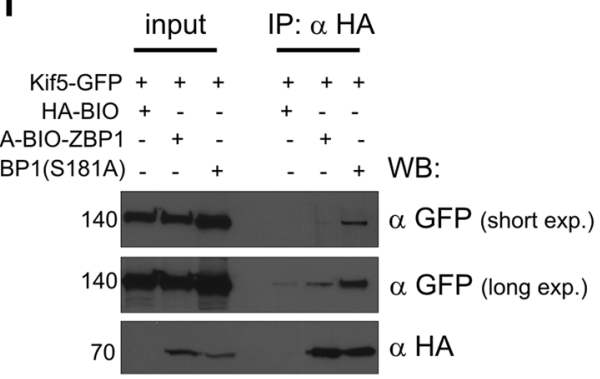

Figure 5. Non-phosphorylated ZBP1 granules are more motile. (a) Representative kymographs showing motility of EGFP-ZBP1 and EGFP-ZBP $1_{\mathrm{S} 181 \mathrm{~A}}$ granules in dendrites. $(\mathbf{b}, \mathbf{c}, \mathbf{d})$ Relative numbers of ZBP1 and $Z B P 1_{S 181 \mathrm{~A}}$ granule movements. Granule movements were quantified, normalized per $10 \mu \mathrm{m}$ of the dendrite length, and then normalized to the mean number of anterograde movements in the wildtype ZBP1 variant for each experiment, respectively. The data in the columns are expressed as a ratio to the mean number of movements. (e) Granule velocity was normalized to the mean anterograde velocity in the wildtype ZBP1 variant for each experiment, respectively. ${ }^{*} * p<0.001,{ }^{*} p<0.1$ (Student's $t$-test). The data were analyzed from five neurons per group per experiment, with three independent experiments. Total raw number of directed anterograde or retrograde movements per group per experiment ranged from 15 to 57, and the number of oscillatory anterograde or retrograde movements per group per experiment ranged from 99 to 245 (for complete data see Table S1). The data are expressed as mean \pm SD. (f) Western blot analysis of KIF5AGFP bound to HA-BIO-ZBP1 or HA-BIO-ZBP1 $1_{\mathrm{S} 181 \mathrm{~A}}$ immunoprecipitated from Neuro2a cells transfected as indicated. Cropped blots are presented (for full length blots see Supplementary Information).

$\mathrm{ZBP} 1_{\mathrm{S} 181 \mathrm{~A}}$ transfected to cultured in vitro hippocampal neurons of $I M P 1^{-1-}$ mice still formed granules, some of which colocalized with $\beta$-actin mRNA (Supplementary Fig. S7), suggesting that the capability of ZBP $1_{S 181 \mathrm{~A}}$ to bind mRNA could not be completely lost. However, in RNA-IP performed from Neuro2a cells transfected with $\mathrm{ZBP} 1$ or $\mathrm{ZBP} 1_{\mathrm{S} 181 \mathrm{~A}}$, we observed that $\mathrm{S} 181 \mathrm{~A}$ substitution resulted in a $50 \%$ decrease in the quantity of $\beta$-actin mRNA in the ZBP1-bound mRNA pool when compared to wildtype ZBP1 (Supplementary Fig. S7). Altogether, we concluded that while there was less $\beta$-actin mRNA bound to $Z B P 1_{S 181 \mathrm{~A}}$ its RNA binding capacity was not the primary reason of the altered $\mathrm{ZBP} 1_{\mathrm{S} 181 \mathrm{~A}}$ distribution in the dendrites.

Lack of Ser181 phosphorylation affects ZBP1 mobility and KIF5A binding. To examine whether the aberrant distribution of $Z B P 1_{S 181 \mathrm{~A}}$ results from a change in the motility of $\mathrm{ZBP} 1$-containing granules, we live-imaged neurons that overexpressed EGFP-ZBP1 or EGFP-ZBP1 $1_{\mathrm{S} 181 \mathrm{~A}}$. To visualize granule movement, we transfected DIV8-9 neurons with wildtype EGFP-ZBP1 or its S181A mutant and recorded time-lapse images 10-14h after transfection. The movies were converted into kymographs, and all single movements were counted. All of the movements were divided into anterograde and retrograde, then classified as either directed $(>2.5 \mu \mathrm{m})$ or oscillatory $(<2.5 \mu \mathrm{m})$. The total number of movements per neuron was normalized per $10 \mu \mathrm{m}$ of the dendrite and then to the mean number of anterograde movements in the wildtype variant in each experiment, respectively, to account for the variability of transfection. As shown in Fig. 5, ZBP1 $1_{\mathrm{S} 181 \mathrm{~A}}$ granules exhibited significantly more directed movements in the anterograde direction compared with the wildtype variant. At the same time, the velocity of retrograde directed movements was significantly elevated. In the case of the oscillatory movements, $\mathrm{ZBP} 1_{\mathrm{S} 181 \mathrm{~A}}$ granules were more motile in both directions, anterograde and retrograde. Consistent with this observation, we found more molecular motor KIF5A-GFP precipitated with ZBP1 $1_{\mathrm{S} 181 \mathrm{~A}}$ than with wildtype ZBP1 when both proteins were overexpressed in Neuro2a cells (Fig. 5f).

\section{Discussion}

In the present study, we found that ZBP1 is stably phosphorylated in neurons at Ser181 in an mTORC2-dependent manner. We also provided evidence that S181 phosphorylation of ZBP1 is decreased in cells with increased Src activity, likely upon ZBP1 phosphorylation by Src. Further investigation of the functional significance of ZBP1 Ser181 phosphorylation in neurons revealed that this posttranslational modification contributed to proper 
dendritic arborization, dendritic ZBP1 distribution and mobility through the regulation of ZBP1 association with motor protein KIF5. We have also demonstrated that ZBP1 S181 phosphorylation is required, to some extent, for $\beta$-actin mRNA binding.

The first important finding of the present study was that in neurons, similar to non-neuronal cells, ZBP1 is phosphorylated at Ser181 in an mTORC2-dependent manner. Under basal conditions, this phosphorylation was stable. In fact, in neurons, the stability of Ser181 phosphorylation appears to be greater than in RD rhabdomyosarcoma cells ${ }^{14}$ because its decrease requires substantially longer mTORC2 inhibition. In RD cells and mouse embryonic fibroblasts (MEFs), Ser181 phosphorylation is protected by mRNA binding. Although not directly, our data support the existence of such a mechanism also in neurons. For example, we showed that $\mathrm{ZBP} 1_{\mathrm{GXXG}}$, which cannot bind $\mathrm{mRNA}$, is poorly phosphorylated at $\mathrm{S} 181$. We also found that the overexpression of active Src decreased Ser181 ZBP1 phosphorylation. Tyr396 ZBP1 phosphorylation by Src results in the release of ZBP1-bound mRNA, and our data are consistent with the model that was proposed by Dai et al. ${ }^{14}$. Src-driven ZBP1 phosphorylation is locally and dynamically regulated in neurons by extracellular stimuli that control neuronal development (e.g., brain-derived neurotrophic factor) ${ }^{6}$. Thus, the present findings suggest that phosphorylation at Ser181 may undergo local dynamic regulation during different steps of neuronal development (e.g., axonal growth and dendritic arborization). However, as discussed below, our study does not address directly physiological consequences of such local and dynamic dephosphorylation of ZBP1.

Another important finding in the present study was that Ser181 ZBP1 phosphorylation is required for proper dendritic arborization. In contrast, Ser181 dephosphorylation does not appear to be as critical for this process, since ZBP1WT and ZBP1 $1_{\mathrm{S} 181 \mathrm{E}}$ did not present different abilities to rescue the effects of endogenous ZBP1 knockdown. This observation is consistent with the hypothesis that the majority of cellular ZBP1 is phosphorylated at Ser $181^{14}$. However, it raises a legitimate question, namely the way in which insufficient Ser181 ZBP1 phosphorylation disturbs dendritogenesis. Our previous study suggested that ZBP1 contributes to dendritic growth to ensure the accuracy of local $\beta$-actin translation ${ }^{9}$. While our results from Neuro2a cells confirm that the S181A mutant binds less $\beta$-actin mRNA, this difference did not show complete loss of function. Additionally, our preliminary results did not reveal differences in $\beta$-actin mRNA colocalization with wildtype ZBP1 and its Ser 181 mutant that were overexpressed in hippocampal neurons (Supplementary Fig S7 and data not shown). Finally, both wildtype ZBP1 and its Ser 181 mutants were comparably phosphorylated by Src. Thus, while we cannot exclude that both $\beta$-actin mRNA binding and release are influenced by a lack of Ser181 phosphorylation, the effect is not as striking and the altered phenotype of the dendritic tree is not likely to be a result of it. Dai et al. ${ }^{14}$ suggested that Ser181 ZBP1 phosphorylation is essential for the initiation of IGF2 mRNA translation using an internal ribosome entry site (IRES). Thus, one possibility is that in neurons that lack Ser181-phosphorylated ZBP1, only the IRES-driven translation of a subset of proteins that are needed for dendritogenesis is decreased. The regulation of IGF2 translation in neurons and the impact of IGF2 on dendritic arborization remain unknown. Several mRNAs that are transported and locally translated in dendrites can utilize IRES-dependent translation ${ }^{18-20}$. Among these are tropomyosin receptor kinase B (TrkB), fragile X mental retardation protein (FMRP1), microtubule associate protein 2 (MAP2), and $\mathrm{Ca}^{2+} /$ calmodulin-dependent protein kinase II (CamKII $\alpha$ ), which are known regulators of dendritic arborization ${ }^{21}$. However, whether their IRES-dependent translation contributes to this process and can be controlled by ZBP1 remain unknown. Finally, we previously found that during intensive dendritogenesis, unlike in more mature neurons, ZBP1 is not equally distributed and is concentrated at dendritic branching points ${ }^{9}$, suggesting a functional link between this specific distribution pattern and dendritic growth. In the present study, $\mathrm{ZBP} 1_{\mathrm{S} 181 \mathrm{~A}}$ failed to concentrate at dendritic branching points and was more evenly distributed along dendrites, thus resembling the distribution of ZBP1 in neurons that passed the dendritogenesis stage. We hypothesize that Ser181 phosphorylation ensures the accurate spatial distribution of ZBP1 that is needed for efficient dendritic growth and branching. Ser181 phosphorylation may promote enrichment of ZBP1 at dendritic branch points and augment the locally translated pool of $\beta$-actin mRNA. Previous work has shown that axonal synthesis of $\beta$-actin supports axon branching of sensory neurons in vitro and in vivo ${ }^{22}$. It has also been demonstrated that axonal branch points are hotspots of local protein synthesis, and they are enriched in ZBP1 and stalled mitochondria, the latter one being critical for local protein synthesis ${ }^{23}$. Curiously, in addition to ZBP1, dendritic branch points are also enriched in mitochondria ${ }^{24}$ as well as translational machinery ${ }^{25}$, suggesting some parallels between dendrite and axon branch points. Therefore, while the role of potential protein synthesis in dendritic branch points remains unclear and ZBP1 S181 phosphorylation in axonal branching has not been investigated, the results of our study suggest a potential mechanisms and provide motivation for future work on shared mechanisms between the dendrite and axon.

How does Ser181 phosphorylation regulate proper dendritic ZBP1 distribution? In this work, we found that $\mathrm{ZBP} 1_{\mathrm{S} 181 \mathrm{~A}}$ moved more than its wildtype counterpart. The greatest difference was observed in the number of movements, whereas the average velocity of the movements of the non-phosphorylated mutant was significantly greater but not as striking. This essentially means that $\mathrm{ZBP} 1_{\mathrm{S} 181 \mathrm{~A}}$-containing granules do not stop as often, suggesting that the increase in motility in the S181A variant was attributable to a disturbance in the mechanism that is responsible for granule docking (Supplementary Fig. S7). Consistent with this, a greater proportion of $\mathrm{ZBP} 1_{\mathrm{S}_{181 \mathrm{~A}}}$-containing granules remained mobile and bound to KIF5A. MyoVa is one example of a protein that can effectively prevent ZBP1 motility in axons ${ }^{26}$. Inhibition of MyoVa resulted in release of ZBP1 from actin and increases motility on microtubules ${ }^{26}$. Thus, lack of Ser 181 phosphorylation may decrease its anchoring to actin at branch points. Alternatively, lack of Ser181 phosphorylation could directly increase its interaction with microtubule motors for transport in the dendrite (e.g., KIF5A, dynein, and KIF11) ${ }^{27,28}$ and/or interactions with other scaffolds (e.g., huntingtin and HAP1) ${ }^{28}$, leading to lower capability of ZBP1 to enter stationary state at the correct destination. Regardless of the exact molecular mechanism enforcing more uniform $\mathrm{ZBP} 1_{\mathrm{S} 181 \mathrm{~A}}$ distribution in dendrites, loss of proper ZBP1 localization likely impairs the discrete pattern of local protein synthesis, required for proper dendritogenesis. 
The present work focused on the role of ZBP1 S181 phosphorylation by examining the consequences of its absence. Our results indicate that phosphorylation of ZBP1 at S181 is needed for its full capacity to bind $\beta$-actin mRNA and accurate mobility of ZBP1-containing granules (or their ability to enter a stationary state needed for proper local mRNA storage; Supplementary Fig. S7). In fact, it is possible that improper ZBP1 mobility results from improper granule formation and/or mRNA loading. However, we can not exclude that these two phenomena are independent. Although S181 phosphorylation is quite stable, our data imply that under some circumstances (e.g. Y396 phosphorylation by Src, RNA release) this serine may undergo physiological dephosphorylation. However, due to the nature of our approach we can only speculate what could be the role of it. Since this dephosphorylation likely occurs as a result of RNA release, which ends the life cycle of a granule, it could simply be the outcome of the disintegration of the granule. Nevertheless, it is also possible that the changed mobility and RNA binding capability, is a result of the dephosphorylation, and it prevents ZBP1, from immediate resilencing of newly released mRNA (Supplementary Fig. S7). However, further study is needed to address the physiological role of dephosphorylation of ZBP1 S181.

\section{Methods}

DNA constructs. The following plasmids were described previously: pGW1-HA ${ }^{29}$, pSuper ${ }^{30}$, pSuper-shRaptor\# $1^{16}$, pSuper-shRictor $\# 2^{16}$, pEGFP-ZBP $1^{8}$, pSuper-ZBP1sh\# $1^{9}$, pEGFP-ZBP $1_{\mathrm{GXXG}}{ }^{9}$, pEGFP-ZBP $1_{\mathrm{Y} 396 \mathrm{~F}}{ }^{9}, \mathrm{pEGFP}-Z B P 1^{*} 1^{9}, \beta$-actin-mRFP ${ }^{31}, \mathrm{pSGT}-\mathrm{Src}^{5}, \mathrm{pSGT}-\operatorname{Src}_{\mathrm{CA}}(\mathrm{K} 239 \mathrm{Q}, \mathrm{P} 250 \mathrm{G})^{5}, \mathrm{pcD}$ NA-Src $\mathrm{KD}_{\mathrm{KD}}(\mathrm{Y} 416 \mathrm{~F})^{5}$, pEGFPC2-BIO ${ }^{32}$, HA-BirA ${ }^{32}$, and tdTomato ${ }^{33}$. pEGFP-ZBP1-P2A-tdTomato was constructed based on pEGFP-ZBP1, in which the GFP stop codon was exchanged with sequences that encoded the P2A signal followed by tdTomato. The change of ZBP1 (or ZBP1*1) Ser181 to alanine (A) or glutamic acid (E) was performed using the Quick Change Site Mutagenesis System (Stratagene, La Jolla, CA, USA). To change Ser181 to A, the following primers were used: 5'-CCCAGGCAGGGGGCACCGGTAGCAG-3' (forward) and 5'-CTGCTACCGGTGCCCCCTGCCTGGG-3' (reverse). The following primers were used for S181E substitution: 5'-CCCAGGCAGGGGGAACCGGTAGCAG-3' (forward) and 5'-CTGCTACCGGTTCCCCCTGCCTGGG-3' (reverse). Substitution of Y396 to F was performed as described previously ${ }^{9}$. Bio-Thrombin-HA plasmid was generated by changing the GFP coding sequence of pEGFPC2-BIO to a sequence that encoded the thrombin recognition site followed by an HA tag and was a kind gift from Dr. Swiech. Next, Bio-Thrombin-HA-ZBP1 and its mutated derivatives were constructed by the polymerase chain reaction (PCR) subcloning of the ZBP1 (or its mutants) coding sequence to EcoRI/SalI sites of Bio-Thrombin-HA. pGW1-Src ${ }^{\mathrm{WT}}-\mathrm{HA}$, pGW1-Src ${ }^{\mathrm{CA}}-\mathrm{HA}$, and pGW1-Src ${ }^{\mathrm{KD}}-\mathrm{HA}$ were obtained by subcloning cDNA-encoding Src and its mutants that were obtained by PCR $\left(5^{\prime}\right.$-GCAGATCTATGGGGAGCAGCAAGAGCAAGC- $3^{\prime}$ and $5^{\prime}$-GGGAATTCCTATAGGTTCTCTCCAGGCTGG-3') in BglII/EcoRI sites of the GW1-HA plasmid. $\beta$-actin-KIF5A-GFP plasmid was a kind gift from Drs. Lipka and Hoogenraad.

Antibodies and drugs. The commercially available primary antibodies that were used in the present study are listed in Table 1. Rabbit anty-P-ZBP1(Tyr396) antibody was described previously ${ }^{6}$. Cross-affinity purified rabbit polyclonal anti-ZBP1(S181) was produced by Eurogentec (Liege, Belgium) against PRQGS*PVAAGA peptide. Both were used at a 1:100 dilution for Western blot. Horseradish peroxidase-conjugated secondary antibodies and IRDye $^{\circledR}$ 680RD anti-mouse and IRDye ${ }^{\circledR} 800 \mathrm{CW}$ were obtained from Jackson ImmunoResearch (West Grove, PA) and LI-COR Biosciences (Lincoln, NE), respectively. INK128 was purchased from Selleckchem (Munich, Germany). Cycloheximide and alkaline phosphatase (FastAP), were obtained from Sigma-Aldrich (St. Louis, MO) and Thermo Scientific (Waltham, MA), respectively.

Primary neuron cultures and transfection. Primary cultures of rat hippocampal and cortical neurons were prepared as described previously ${ }^{9}$ in accordance with the institutional guidelines of the First Local Ethics Committee in Warsaw (decision for experimental protocol no. 205/2011), which are in compliance with the European Community Council Directive (86/609/EEC). Cells were transfected on the indicated DIV using Lipofectamine 2000 or nucleofected on DIV0 as described previously ${ }^{9}$. Primary cultures of hippocampal neurons of $I M P 1^{-1-}$ mice were prepared from E17 embryos obtained by crossing $I M P 1^{+1-}$ mice as described ${ }^{34}$ and in accordance with the institutional guidelines of Emory University (protocol approved by Emory University IACUC committee [GJB]). DIV7 cells were transfected using Lipofectamine2000.

Neuro2A culture, transfection and drug treatment. Neuro2a cells (ATCC, Manassas, VA, USA) were grown in Dulbecco's Modified Eagle Medium that contained $10 \%$ fetal bovine serum and $1 \%$ penicillin-streptomycin (all from Sigma-Aldrich) at $37^{\circ} \mathrm{C}$ in a $5 \% \mathrm{CO}_{2}$ atmosphere. The cells were transfected using JetPEI (PolyPlus, Illkirch, France) at a 3:1 PEI:DNA ratio according to the manufacturer's instructions. Eighteen hours after transfection, the cells were treated with cycloheximide $(10 \mu \mathrm{g} / \mathrm{ml})$ for $0.5,1,3,6$ or 24 hours. The control cells were treated with vehicle ( $98 \%$ ethanol).

In vitro phosphatase assay. In vitro phosphatase assay is described in Supplementary Materials and Methods.

Avi-tag pulldown and immunoprecipitation. Biotinylated forms of proteins were produced in the Neuro2a cell line. These cells were transfected with a plasmid that encoded ZBP1 or its mutants with an avi-tag and BirA plasmid that encoded the biotin ligase ${ }^{32}$. Eighteen hours after transfection, the cells were placed in ice-cold PBS, centrifuged at $8,000 \times g$ for $3 \mathrm{~min}$, and lysed for $10 \mathrm{~min}$ in lysis buffer $(20 \mathrm{mM}$ Tris [pH 7.5], $100 \mathrm{mM}$ $\mathrm{NaCl}$, and $0.5 \% \mathrm{NP}-40$ ) supplemented with protease and phosphatase inhibitors. The cell lysate was clarified by centrifugation at $18,000 \times g$ at $4^{\circ} \mathrm{C}$ for $20 \mathrm{~min}$ and incubated for $1.5 \mathrm{~h}$ at $4{ }^{\circ} \mathrm{C}$ with streptavidin-coated magnetic beads (Dynabeads M-280, Invitrogen). After incubation, the beads were washed five times with lysis buffer, and bound proteins were recovered by boiling the samples with $1 \times$ Laemmli buffer. 


\begin{tabular}{|c|c|c|c|c|}
\hline Antigen & Manufacturer & Cat. number & Host & Application \\
\hline Akt & $\begin{array}{l}\text { Cell Signaling } \\
\text { Technology Danvers, } \\
\text { MA }\end{array}$ & 4298 & mouse & WB 1:2000 \\
\hline phospho-Akt (Ser473) & $\begin{array}{l}\text { Cell Signaling } \\
\text { Technology, }\end{array}$ & 4060 & rabbit & WB 1:1000 \\
\hline$\beta$-actin & $\begin{array}{l}\text { Sigma-Aldrich, Saint } \\
\text { Louis, MO }\end{array}$ & A2228 & mouse & IF $1: 1000$ \\
\hline GAPDH & $\begin{array}{l}\text { Synaptic Systems, } \\
\text { Goettingen, Germany }\end{array}$ & 247002 & rabbit & WB $1: 1000$ \\
\hline GAPDH & $\begin{array}{l}\text { EMD Millipore, } \\
\text { Billerica, MA }\end{array}$ & MAB374 & mouse & WB 1:1000 \\
\hline GFP & MBL, Woburn, MA & 598 & rabbit & $\begin{array}{l}\text { IF 1:1000 WB } \\
1: 3000\end{array}$ \\
\hline GFP & Sigma Aldrich & $\begin{array}{l}11814460 \\
001\end{array}$ & mouse & $\begin{array}{l}\text { RNA-IP: } 15 \mu \mathrm{g} / \\
\text { sample }\end{array}$ \\
\hline HA & Sigma-Aldrich & $\begin{array}{l}11867423 \\
001\end{array}$ & rat & WB $1: 1000$ \\
\hline IGF2BP1 (ZBP1) & MBL & RN007P & rabbit & WB 1:1000 \\
\hline IGF2BP1 (ZBP1) & MBL & RN001M & mouse & WB 1:1000 \\
\hline IMP1 (ZBP1) & $\begin{array}{l}\text { SantaCruz } \\
\text { Biotechnology, Dallas, } \\
\text { TX }\end{array}$ & 21026 & goat & WB 1:1000 \\
\hline IMP1 (ZBP1) & $\begin{array}{l}\text { Cell Signaling } \\
\text { Technology }\end{array}$ & 8482 & rabbit & WB 1:1000 \\
\hline Raptor & $\begin{array}{l}\text { Cell Signaling } \\
\text { Technology }\end{array}$ & 2280 & rabbit & WB 1:1000 \\
\hline Rictor & $\begin{array}{l}\text { Cell Signaling } \\
\text { Technology }\end{array}$ & $2114 \mathrm{~S}$ & rabbit & WB 1:1000 \\
\hline S6 & $\begin{array}{l}\text { Cell Signaling } \\
\text { Technology }\end{array}$ & 2217 & rabbit & WB 1:1000 \\
\hline phospho-S6 (Ser235/236) & $\begin{array}{l}\text { Cell Signaling } \\
\text { Technology }\end{array}$ & $4858 \mathrm{~L}$ & rabbit & WB 1:1000 \\
\hline $\mathrm{v}-\mathrm{Src}$ & $\begin{array}{l}\text { EMD Millipore, } \\
\text { Billerica, MA }\end{array}$ & OP07 & mouse & WB 1:1000 \\
\hline mRFP (tdTomato) & $\begin{array}{l}\text { Evrogen, Moscow, } \\
\text { Russia }\end{array}$ & AB233 & rabbit & WB 1:1000 \\
\hline$\alpha$-tubulin & Sigma-Aldrich & T5168 & mouse & WB 1:5000 \\
\hline
\end{tabular}

Table 1. Primary antibodies used for the study. WB - Western blot, IF - immunofluorescence.

For immunprecipitation Neuro2a cells were transfected with plasmids encoding Kif5a-GFP and Bio-HA (negative control), ZBP1-Bio-HA, or ZBP1 $1_{\mathrm{S} 181}$-Bio-HA. Forty-eight hours after transfection, the cells were collected in ice-cold TBS-K $(20 \mathrm{mM}$ Tris [pH 7.5], $100 \mathrm{mM} \mathrm{NaCl})$, centrifuged at $4,000 \times g$ for $10 \mathrm{~min}$, and lysed for $20 \mathrm{~min}$ in lysis buffer (50 mM Tris [pH 7.5], $150 \mathrm{mM} \mathrm{NaCl}, 0,1 \%$ Triton X-100, $2 \mathrm{mM} \mathrm{EDTA,} 2 \mathrm{mM} \mathrm{MgCl}_{2}$ ) supplemented with $2 \mathrm{mM}$ ATP. The cell lysate was cleared by centrifugation at $13,000 \times \mathrm{g}$ at $4{ }^{\circ} \mathrm{C}$ for $20 \mathrm{~min}$ and incubated overnight with magnetic beads covered with anti-HA antibody (Pierce ${ }^{\mathrm{TM}}$ Anti-HA Magnetic Beads, ThermoFisher). After incubation, the beads were washed four times with lysis buffer without ATP, and boiled in $1 \times$ Laemmli buffer.

RNA co-immunoprecipitation. Immunoprecipitation was performed as before, with modifications (Janusz et al., 2013) ${ }^{35}$. Briefly, Neuro2A cells were transfected with EGFP-ZBP1 or EGFP-ZBP $1_{S 181 \mathrm{~A}}$ plasmid. Next day, cells were collected, washed with PBS and suspended in $1 \mathrm{ml}$ of a buffer containing $140 \mathrm{mM} \mathrm{KCl}, 1.5 \mathrm{mM}$ $\mathrm{MgCl}_{2}, 20 \mathrm{mM}$ Tris- $\mathrm{HCl}$ ( $\mathrm{pH}$ 7.4), 0.5\% Nonidet P-40, $0.5 \mathrm{mM}$ dithiothreitol, $100 \mathrm{U} / \mathrm{ml}$ RiboLock (Thermo Scientific) and protease inhibitors. Extracts $(800 \mu \mathrm{g}$ of total protein) were precleared with $60 \mu \mathrm{l}$ of Dynabeads Protein G (Invitrogen) for $2.5 \mathrm{~h}$. Afterward, $1 / 10$ of each supernatant was saved as input fraction for WB and RNA isolation. Protein-RNA complexes were precipitated overnight in $4{ }^{\circ} \mathrm{C}$ with $60 \mu$ of Dynabeads Protein G, conjugated with either mouse anti-GFP antibody (Sigma-Aldrich) or normal mouse IgG (Sigma-Aldrich). Next, $1 / 6$ of the beads was collected and boiled with $1 \times$ Laemmli buffer for WB. The remaining beads were suspended in $500 \mu \mathrm{l} \mathrm{TRIzol}$ (Thermo Scientific) for $5 \mathrm{~min}$. Then, TRIzol was collected and RNA was isolated. For the quantitative real-time (qRT)-PCR, RNA was suspended in $11 \mu$ of $\mathrm{H}_{2} \mathrm{O}$, then its concentration was determined with NanoDrop (Thermo Scientific). Reverse-transcription was performed with High Capacity cDNA Reverse Transcription Kit (Applied Biosystems, Foster City, CA), according to manufacturer's instruction.

Quantitative real time PCR. For the RT-qPCR, Taqman probes for $\beta$-actin and the control gene USP16 were used (Mm01344526_m1, Mm01205647_g1; Applied Biosystems). Fold enrichment in the IP fraction was calculated for both mRNAs according to the standard curve method, using Input ZBP1 WT cDNA as a standard. Results were corrected for the non-specific background in the IgG fraction and ratios of $\beta$-actin and USP16 mRNA were calculated. To account for the difference in the transfection levels ratios were normalized to the ZBP1 WT IP fraction. 
Western blot. Qualitative and quantitative Western blot analyses were performed as described previously ${ }^{36}$.

Imaging of fixed cells and image analysis. The acquisition of confocal images of fixed neurons and morphometric analysis were performed as described previously ${ }^{9}$. Dendritic distribution of overexpressed EGFP-ZBP1 and its mutants was analyzed as previously ${ }^{9}$ with additional normalization step. Briefly, images of cells were obtained using a Zeiss LSM710 NLO laser confocal microscope with a $100 \times 1.3$ objective. Images were acquired in $\mathrm{z}$-series $(0.5 \mu \mathrm{m}$ steps), with identical acquisition settings for all neurons. For analysis, $\mathrm{z}$-series were rendered using maximum intensity projections, exported into TIFF format, and analyzed using MetaMorph software. Profiles of EGFP-ZBP1 and TdTomato fluorescence along the dendrites were made using the Linescan function, with the scan width set to 6 pixels. For each pixel EGFP fluorescence was standardized to fluorescence of TdTomato. Next, branching sites were indicated manually, based on the TdTomato imaging of dendrites and the mean standardized intensities of EGFP fluorescence at (R segments) and between ( $\mathrm{D}$ segments) the branching sites were calculated using OpenOffice Calc software. D and R segments were not of the same length or area.

Live imaging of hippocampal neurons in culture. For live imaging, rat primary hippocampal neurons were cultured on glass-bottom multi-well plate dishes (12-well, no. 1.0 coverslip, $14 \mathrm{~mm}$ glass diameter; MatTek, Ashland, MA, USA), pre-cleaned with $1 \mathrm{M} \mathrm{HCl}$ according to the manufacturer's specifications, and then coated with laminin and poly-L-lysine ${ }^{9}$. Neurons were transfected on DIV7-8 with pEGFP-ZBP1 or pEGFP-ZBP $1_{\mathrm{S}_{181 A}}$. Time-lapse images ( 135 frames in $0.444 \mathrm{~s}$ intervals) of neurons that expressed EGFP-ZBP1 or EGFP-ZBP $1_{\mathrm{S} 181 \mathrm{~A}}$ were collected 10-14 h after transfection. Image analysis was performed using ImageJ software. All stack images were first corrected for X-Y drift. Afterward, to remove immobile background foci, the averaged image was subtracted from the image stack. The region of interest (ROI) for all dendrites in focus that were longer than $15 \mu \mathrm{m}$ were converted to kymographs $(\mathrm{x}=$ dendrite length, $\mathrm{y}=$ time). All single movements were counted and divided into anterograde or retrograde based on their $\mathrm{x}-\mathrm{y}$ slope, then classified as either directed $(>2.5 \mu \mathrm{m})$ or oscillatory $(<2.5 \mu \mathrm{m})$.

Statistical analysis. The exact numbers of cells $(n)$ and culture batches $(N)$ that were examined and the statistical tests that were used for the respective experiments are indicated in the figure legends. The statistical analyses were performed using Prism software (GraphPad, San Diego, CA).

\section{References}

1. Swanger, S. A. \& Bassell, G. J. Dendritic protein synthesis in the normal and diseased brain. Neuroscience 232, 106-127, doi:10.1016/j. neuroscience.2012.12.003 (2013).

2. Jung, H., Gkogkas, C. G., Sonenberg, N. \& Holt, C. E. Remote control of gene function by local translation. Cell 157, 26-40, doi:10.1016/j.cell.2014.03.005 (2014).

3. Bramham, C. R. \& Wells, D. G. Dendritic mRNA: transport, translation and function. Nat Rev Neurosci 8, 776-89, doi:10.1038/ nrn2150 (2007)

4. Kiebler, M. A. \& Bassell, G. J. Neuronal RNA granules: movers and makers. Neuron 51, 685-90, doi:10.1016/j.neuron.2006.08.021 (2006).

5. Huttelmaier, S. et al. Spatial regulation of beta-actin translation by Src-dependent phosphorylation of ZBP1. Nature 438, 512-5, doi:10.1038/nature04115 (2005).

6. Sasaki, Y. et al. Phosphorylation of zipcode binding protein 1 is required for brain-derived neurotrophic factor signaling of local beta-actin synthesis and growth cone turning. J. Neurosci 30, 9349-9358, doi:10.1523/JNEUROSCI.0499-10.2010 (2010).

7. Narayanan, U. et al. S6K1 phosphorylates and regulates fragile X mental retardation protein (FMRP) with the neuronal protein synthesis-dependent mammalian target of rapamycin (mTOR) signaling cascade. J. Biol. Chem. 283, 18478-18482, doi:10.1074/jbc. C800055200 (2008).

8. Eom, T., Antar, L. N., Singer, R. H. \& Bassell, G. J. Localization of a beta-actin messenger ribonucleoprotein complex with zipcodebinding protein modulates the density of dendritic filopodia and filopodial synapses. J Neurosci 23, 10433-44 (2003).

9. Perycz, M., Urbanska, A. S., Krawczyk, P. S., Parobczak, K. \& Jaworski, J. Zipcode binding protein 1 regulates the development of dendritic arbors in hippocampal neurons. J Neurosci 31, 5271-85, doi:10.1523/JNEUROSCI.2387-10.2011 (2011).

10. Tiruchinapalli, D. M. et al. Activity-dependent trafficking and dynamic localization of zipcode binding protein 1 and beta-actin mRNA in dendrites and spines of hippocampal neurons. J Neurosci 23, 3251-61 (2003).

11. Zhang, H. L. et al. Neurotrophin-induced transport of a beta-actin mRNP complex increases beta-actin levels and stimulates growth cone motility. Neuron 31, 261-75, doi:10.1016/S0896-6273(01)00357-9 (2001).

12. Leung, K. M. et al. Asymmetrical beta-actin mRNA translation in growth cones mediates attractive turning to netrin-1. Nat Neurosci 9, 1247-56, doi:10.1038/nn1775 (2006).

13. Ceci, M. et al. RACK1 is a ribosome scaffold protein for $\beta$-actin mRNA/ZBP1 complex. PloS One 7, e35034, doi:10.1371/journal. pone.0035034 (2012)

14. Dai, N., Christiansen, J., Nielsen, F. C. \& Avruch, J. mTOR complex 2 phosphorylates IMP1 cotranslationally to promote IGF2 production and the proliferation of mouse embryonic fibroblasts. Genes Dev 27, 301-12, doi:10.1101/gad.209130.112 (2013).

15. Hansen, T. V. O. et al. Dwarfism and impaired gut development in insulin-like growth factor II mRNA-binding protein 1-deficient mice. Mol. Cell. Biol. 24, 4448-4464, doi:10.1128/MCB.24.10.4448-4464.2004 (2004).

16. Urbanska, M., Gozdz, A., Swiech, L. J. \& Jaworski, J. Mammalian target of rapamycin complex 1 (MTORC1) and 2 (MTORC2) control the dendritic arbor morphology of hippocampal neurons. J Biol Chem 287, 30240-56, doi:10.1074/jbc.M112.374405 (2012).

17. Skałecka, A. et al. mTOR kinase is needed for the development and stabilization of dendritic arbors in newly born olfactory bulb neurons. Dev. Neurobiol. 76, 1308-27, doi:10.1002/dneu.22392 (2016).

18. Pinkstaff, J. K., Chappell, S. A., Mauro, V. P., Edelman, G. M. \& Krushel, L. A. Internal initiation of translation of five dendritically localized neuronal mRNAs. Proc. Natl. Acad. Sci. USA 98, 2770-2775, doi:10.1073/pnas.051623398 (2001)

19. Dobson, T., Minic, A., Nielsen, K., Amiott, E. \& Krushel, L. Internal initiation of translation of the TrkB mRNA is mediated by multiple regions within the $5^{\prime}$ leader. Nucleic Acids Res. 33, 2929-2941, doi:10.1093/nar/gki605 (2005).

20. Chiang, P. W., Carpenter, L. E. \& Hagerman, P. J. The $5^{\prime}$-untranslated region of the FMR1 message facilitates translation by internal ribosome entry. J. Biol. Chem. 276, 37916-37921, doi:10.1074/jbc.M105584200 (2001).

21. Urbanska, M., Blazejczyk, M. \& Jaworski, J. Molecular basis of dendritic arborization. Acta Neurobiol Exp Wars 68, 264-88 (2008).

22. Donnelly, C. J. et al. Axonally synthesized $\beta$-actin and GAP-43 proteins support distinct modes of axonal growth. J. Neurosci 33, 3311-3322, doi:10.1523/JNEUROSCI.1722-12.2013 (2013).

23. Spillane, M., Ketschek, A., Merianda, T. T., Twiss, J. L. \& Gallo, G. Mitochondria coordinate sites of axon branching through localized intra-axonal protein synthesis. Cell Rep. 5, 1564-1575, doi:10.1016/j.celrep.2013.11.022 (2013). 
24. Faits, M. C., Zhang, C., Soto, F. \& Kerschensteiner, D. Dendritic mitochondria reach stable positions during circuit development. eLife 5, e11583, doi:10.7554/eLife.11583 (2016).

25. Bartlett, W. P. \& Banker, G. A. An electron microscopic study of the development of axons and dendrites by hippocampal neurons in culture. I. Cells which develop without intercellular contacts. J. Neurosci 4, 1944-1953 (1984).

26. Nalavadi, V. C. et al. Regulation of zipcode binding protein 1 transport dynamics in axons by myosin Va. J. Neurosci 32, 15133-15141, doi:10.1523/JNEUROSCI.2006-12.2012 (2012).

27. Song, T. et al. Specific interaction of KIF11 with ZBP1 regulates the transport of $\beta$-actin mRNA and cell motility. J. Cell Sci. 128, 1001-1010, doi:10.1242/jcs.161679 (2015).

28. Ma, B. et al. Huntingtin mediates dendritic transport of $\beta$-actin mRNA in rat neurons. Sci. Rep. 1, 140, doi:10.1038/srep00140 (2011).

29. Pak, D.T., Yang, S., Rudolph-Correia, S., Kim, E. \& Sheng, M. Regulation of dendritic spine morphology by SPAR, a PSD-95associated RapGAP. Neuron. Aug 2, 31(2), 289-303 (2001).

30. Brummelkamp, T. R., Bernards, R. \& Agami, R. A system for stable expression of short interfering RNAs in mammalian cells. Science 296, 550-3, doi:10.1126/science.1068999 (2002).

31. Hoogenraad, C. C. et al. Liprinalphal degradation by calcium/calmodulin-dependent protein kinase II regulates LAR receptor tyrosine phosphatase distribution and dendrite development. Dev Cell 12, 587-602, doi:10.1016/j.devcel.2007.02.006 (2007).

32. de Boer, E. et al. Efficient biotinylation and single-step purification of tagged transcription factors in mammalian cells and transgenic mice. Proc Natl Acad Sci USA 100, 7480-5, doi:10.1073/pnas.1332608100 (2003).

33. Shaner, N. C. et al. Improved monomeric red, orange and yellow fluorescent proteins derived from Discosoma sp. red fluorescent protein. Nat Biotechnol 22, 1567-72, doi:10.1038/nbt1037 (2004).

34. Welshhans, K. \& Bassell, G. J. Netrin-1-induced local $\beta$-actin synthesis and growth cone guidance requires zipcode binding protein 1. J. Neurosci 31, 9800-9813, doi:10.1523/JNEUROSCI.0166-11.2011 (2011).

35. Janusz, A., et al. The Fragile X mental retardation protein regulates matrix metalloproteinase 9 mRNA at synapses. J Neurosci. 2013 Nov 13,33(46), 18234-41, doi:10.1523/JNEUROSCI.2207-13 (2013).

36. Malik, A. R. et al. Tuberous sclerosis complex neuropathology requires glutamate-cysteine ligase. Acta Neuropathol. Commun. 3, 48, doi:10.1186/s40478-015-0225-z (2015).

\section{Acknowledgements}

We thank Dr. Huttelmaier for Src-CA and DN mutant cDNA, Dr. Swiech for Bio-Thrombin-HA plasmid and Drs. Lipka and Hoogenraad for plasmid encoding GFP-tagged full length rat KIF5a. We thank Monika Matuszczyk and Alina Zielinska for technical help. This research was supported by the Polish National Science Centre (2011/01/N/NZ3/05405) to A.S.U. and an ICEGB grant to J.J., A.J.-K. and K.S. were supported by a National Science Centre Sonata-Bis grant (2012/07/E/NZ3/00503). M.U. was supported by a Polish Ministry of Science and Higher Education Iuventus Plus grant (IP2012 037872). J.J., K.S., and M.U. are also recipients of the Foundation for Polish Science "Mistrz" Professorial Subsidy and Fellowship, respectively.

\section{Author Contributions}

A.S.U., A.J.-K., G.B., and J.J. designed the experiments. A.S.U., A.J.-K., K.S., A.H., M.P., L.S., M.U., and J.J. conducted the experiments. G.B. and A.H. contributed unique reagents. A.S.U., A.J.-K., and J.J. wrote the manuscript.

\section{Additional Information}

Supplementary information accompanies this paper at doi:10.1038/s41598-017-01963-2

Competing Interests: The authors declare that they have no competing interests.

Publisher's note: Springer Nature remains neutral with regard to jurisdictional claims in published maps and institutional affiliations.

(c) (i) Open Access This article is licensed under a Creative Commons Attribution 4.0 International LY License, which permits use, sharing, adaptation, distribution and reproduction in any medium or format, as long as you give appropriate credit to the original author(s) and the source, provide a link to the Creative Commons license, and indicate if changes were made. The images or other third party material in this article are included in the article's Creative Commons license, unless indicated otherwise in a credit line to the material. If material is not included in the article's Creative Commons license and your intended use is not permitted by statutory regulation or exceeds the permitted use, you will need to obtain permission directly from the copyright holder. To view a copy of this license, visit http://creativecommons.org/licenses/by/4.0/.

(c) The Author(s) 2017 